\title{
OPEN An electrochemical sensor for nanomolar detection of caffeine based on nicotinic acid hydrazide anchored on graphene oxide (NAHGO)
}

\author{
Jemini Jose ${ }^{1}$, Viswanathan Subramanian ${ }^{3}$, Sadasivan Shaji ${ }^{2}$ P. B. Sreeja ${ }^{1 凶}$
}

A simple modified sensor was developed with nicotinic acid hydrazide anchored on graphene oxide (NAHGO), by ultrasonic-assisted chemical route, using hydroxy benzotriazole as a mediator. Structural and morphologies of NAHGO samples were investigated in detail by Fourier-Transform Infrared spectroscopy (FT-IR), Powder X-ray diffraction (P-XRD), Raman spectroscopy, Scanning electron microscopy (SEM), X-ray photoelectron spectroscopy (XPS), and Thermogravimetric analysis (TGA). The detailed morphological examination and electrochemical studies revealed the delaminated sheet with the tube-like structure of NAHGO provided the route for more electroactive surface which influenced the electrooxidation of caffeine with increased current. The electrochemical behaviour of NAHGO on a glassy carbon electrode (GCE) for caffeine detection was demonstrated by employing voltammetric techniques. The influence of scan rate, $\mathrm{pH}$, and concentration on caffeine's peak current was also studied. The NAHGO sensor was employed for the determination of caffeine in imol plus and energy drinks. The detection limit determined was $8.7 \times 10^{-9} \mathrm{M}$, and the best value was reported so far. The results show that NAHGO modified electrodes are one of the best preferences to establish new, efficient, and reliable analytical tools for the detection of caffeine.

Carbon materials have a pivotal role in detecting an unsafe amount of organic and inorganic molecules due to their highly porous structure with large surface area and excellent electronic properties ${ }^{1}$. Graphene oxide (GO) has received much attention among carbon nanomaterials due to its high mechanical strength, thermal, and electrical conductivity ${ }^{2}$. Besides, the carboxyl, hydroxy, and epoxy groups create GO as a desirable platform for further functionalizing molecules ${ }^{3,4}$. Other than functionalization approaches of GO, heteroatom doping is also important since it modifies the properties of GO extensively. Among various heteroatom doping, nitrogen doping is widely utilized to employ doping of GO. This is because of the easiness of manipulating GO to improve more active sites for fuel cell, biosensing, adsorption, supercapacitor, and solar cell applications ${ }^{5,6}$. Nitrogen doping of $\mathrm{GO}$ creates different kinds of nitrogen such as pyrrolic-N, pyridinic- $\mathrm{N}$, and graphitic- $\mathrm{N}$ in the GO lattice, which enrich the electronic properties of $\mathrm{GO}^{7,8}$. It has been reported that nitrogen doping changes the spin density and charge distribution on the surface of GO, with the strong interaction between the $\pi$-system of $\mathrm{GO}$ and the lone pair of electrons of nitrogen ${ }^{9}$. At present, different methods such as plasma treatment, segregation growth, chemical vapour deposition, and arc discharge method have been developed for the synthesis of nitrogen-doped GO. However, the practical applications of these methods are limited due to high and complex reaction temperature conditions ${ }^{6}$. Hence simple organic compounds with amino groups like urea, melamine, glycine, and hydroxyl amine are utilized to achieve nitrogen doping. The integration of nitrogen atoms in the GO framework brings out significant changes in the electronic structure resulting in the reduction of $\mathrm{GO}^{10}$. The incoming molecules' active sites such as diisopropylamine, diaminopyridine, dibenzylamine, phenylenediamine, piperidine, phenylethylamine, 2,4-dinitrophenylhydrazine, Bis-(pththalimidoethyl)-amine, and Tris-(hydroxymethyl) methyl aminoethane generate new covalent bonds with $\mathrm{GO}^{11,12}$. Therefore, in this paper we report the synthesis of

${ }^{1}$ Department of Chemistry, CHRIST (Deemed to be University), Bengaluru, Karnataka 560029, India. ${ }^{2}$ Facultad de Ingeniería Mecánica y Eléctrica, Universidad Autónoma de Nuevo León, 66455 San Nicolás de los Garza, Nuevo León, Mexico. ${ }^{3}$ Department of Industrial Chemistry, Alagappa University, Karaikudi, Tamil Nadu 630003, India. ${ }^{\square}$ email: sreeja.pb@christuniversity.in 
NAHGO by a simple and cost effective ultrasonic-assisted chemical route where the amino group of NAH reacts with the oxygen containing carboxylic acid, hydroxyl, and epoxy groups of GO that brings the nitrogen doping and the simultaneous reduction of GO.

Caffeine (1,3,7-trimethyl xanthine) is one of the most consumed purine alkaloid ingredients. It is a central nervous system stimulant, fatigue alleviator, sharpener of mental function and concentration, taste refresher with a unique taste, antioxidant, booster of metabolism, and associated health benefits ${ }^{13,14}$. It is commonly found in coffee beans, tea leaves, guarana berries, pharmaceutical drugs, processed foods, and energy drinks ${ }^{15}$. The sensible amount of caffeine in medications reduces constipation, depression, and headache. Like all other drugs, caffeine overdose produces anxiety, heartburns, increased blood pressure, seizures, bone mass loss, and cardiovascular diseases ${ }^{16,17}$. On the other hand, the extracts of caffeine act as a pesticide for plants, and it has been considered an indicator for water contamination in sewers ${ }^{18,19}$. Hence, there is a need for detecting caffeine reliably at lower concentrations, especially in common beverages, caffeinated food, and drugs, since the average consumption of caffeine per day is up to $0.4 \mathrm{~g}^{20}$.

Different analytical techniques are currently available to determine caffeine, such as high-performance liquid chromatography (HPLC), UV spectrometry, capillary electrophoresis, spectrophotometry $y^{21-24}$. These analyses are complicated because of the lengthy and time-consuming procedures. Hence the development of highly sensitive, consistent, and fast methods is essential.

The electrochemical method has proved its quality and efficiency for the fabrication of sensing devices ${ }^{25,26}$. It acts as a powerful tool for detecting and monitoring the unsafe amount of organic and inorganic compounds using different carbon materials. Caffeine has already been electrochemically detected with the boron-doped diamond electrode, DNA-functionalised single-walled carbon nanotubes (SWCNT), attapulgite/nafion, gold nanoparticle, gold-chitosan, multiwalled carbon nanotubes (MWCNT)/diamond-like carbon films, glutathione-rGO, etc $^{27-31}$. Hence, we propose a new kind of electrochemical sensor supported by GO and nicotinic acid hydrazide (NAH) to detect caffeine. The synthesised NAHGO was analysed by different spectroscopic and morphological methods and then studied caffeine' electrochemical detection. We found that NAHGO showed catalytic activity for the electro-chemical oxidation of caffeine.

\section{Results and discussion}

Characterizations of NAHGO. The X-ray photoelectron spectrum of the hybrid NAHGO is performed to assess the surface composition and chemical states of the elements present in NAHGO. The broad scan spectrum of GO and NAHGO are displayed in Fig. 1a. GO shows the peaks of C1s and O1s, whereas NAHGO exhibits carbon, nitrogen, and oxygen, with major prominent peaks at 285.12, 401.23, and $533.41 \mathrm{eV}$ corresponds to $\mathrm{C} 1 \mathrm{~s}, \mathrm{~N} 1 \mathrm{~s}$, and $\mathrm{O} 1 \mathrm{~s}$, respectively. The C1s high-resolution spectrum is deconvoluted into four components, as shown in Fig. 1b. The peaks at 284.5, 285.4, 286.1, and $287.6 \mathrm{eV}$ attributed to $\mathrm{C}-\mathrm{C} / \mathrm{C}=\mathrm{C}$ of graphene oxide framework, $\mathrm{C}-\mathrm{N}, \mathrm{C}-\mathrm{O}$, and $\mathrm{HN}-\mathrm{C}=\mathrm{O}$ bonds. The deconvoluted peaks of $\mathrm{O} 1 \mathrm{~s}$ at 531.5 and $532.9 \mathrm{eV}$, as indicated in Fig. $1 \mathrm{c}$, are due to the characteristic $\mathrm{C}-\mathrm{O}, \mathrm{C}=\mathrm{O} / \mathrm{C}-\mathrm{N}-\mathrm{O}$ of $\mathrm{NAHGO}^{32,33}$. The individual peaks of N1s at 399.6 and $400.8 \mathrm{eV}$, which are exhibited in Fig. $1 \mathrm{~d}$, attribute to the presence of pyridinic $\mathrm{N}$ and pyrrolic $\mathrm{N}$, respectively. There is a high peak at $500 \mathrm{eV}$ due to the auger line of sodium $\left(\mathrm{Na} 2 \mathrm{~s} \text { or } \mathrm{Na}_{\mathrm{KLL}}\right)^{33,34}$. The EDAX data is shown in Fig. S1. The XRD patterns of GO and NAHGO is presented in Fig. 1e. The intense $2 \theta$ peak at $10.7^{\circ}(001)$ diffraction plane of as prepared GO shows an inter-planar distance of $0.83 \mathrm{~nm}$, calculated by Bragg's equation. In NAHGO, $10.7^{\circ}$ of GO is disappeared and shifted to a high $2 \theta$ value, showing the reduction of oxygen containing functional groups and incorporating nitrogen atoms onto graphene surface (XRD of NAH is shown in Fig. S2). The Raman spectra of GO and NAHGO display two major bands, as shown in Fig. 1f. The C=C stretching vibration of $\mathrm{sp}^{2}$ hybridised carbon systems illustrate the $\mathrm{G}$ band at about $1592 \mathrm{~cm}^{-1}$ and D band at $1352 \mathrm{~cm}^{-1} \mathrm{cor}^{-}$ responds to the distortions in the edge-centred band structure of the carbon framework of $\mathrm{GO}^{35}$. In NAHGO, these two peaks shift to $1581 \mathrm{~cm}^{-1}$ and $1341 \mathrm{~cm}^{-1}$, confirming the reduction in hybrid $\mathrm{GO}^{36}$. The intensity ratio $\mathrm{I}_{\mathrm{D}} / \mathrm{I}_{\mathrm{G}}$ of GO and NAHGO are 0.96 and 1.03 , respectively. The increased intensity ratio of $\mathrm{I}_{\mathrm{D}} / \mathrm{I}_{\mathrm{G}}$ in NAHGO attributed to defects present after the functionalization of $\mathrm{GO}^{37}$.

The IR spectra of GO and NAHGO are shown in Fig. 2a. The broad peak at $3375 \mathrm{~cm}^{-1}$ is attributed to the stretching vibration of carboxyl $-\mathrm{OH}$ for $\mathrm{GO}$. The $\mathrm{C}=\mathrm{O}, \mathrm{C}-\mathrm{OH}$, and $\mathrm{C}-\mathrm{O}$ of carboxylic and epoxy groups represent peaks at $1721 \mathrm{~cm}^{-1}, 1215 \mathrm{~cm}^{-1}$, and $1030 \mathrm{~cm}^{-1}$, respectively ${ }^{37-39}$. Three new peaks are observed in the spectra of NAHGO. They are at $1625 \mathrm{~cm}^{-1}$, correspond to the amide carbonyl stretch; $1561 \mathrm{~cm}^{-1}$ is due to the $\mathrm{N}-\mathrm{H}$ of amide band; $1445 \mathrm{~cm}^{-1}$, the $\mathrm{C}-\mathrm{N}$ stretching of amide bond $\mathrm{d}^{40,41}$. This is in accordance with XPS, support the nitrogen doping of GO by nicotinic acid hydrazide. The absorption bands at 2950 and $2900 \mathrm{~cm}^{-1}$ are assigned to the symmetric and asymmetric stretching of $\mathrm{C}-\mathrm{H}$ vibrations ${ }^{41}$. The band at $2100 \mathrm{~cm}^{-1}$ corresponds to $\mathrm{N}=\mathrm{C}=\mathrm{N}$ vibrations ${ }^{42,43}$. Thermal stability of the GO and NAHGO were investigated by TGA and are shown in Fig. 2b. The slow degradation of NAHGO with GO indicates the high stability which is acquired by reducing GO and the presence of nitrogen atoms in NAHGO. The XPS data of GO and NAHGO also supports the same. The GO and NAHGO were examined by SEM to understand more about these compounds' morphology and microstructure. As seen in Fig. 2c-f, GO demonstrated the extended sheet-like structures, and NAHGO results the deformation of GO owing to the amide bond formation and esterification reaction with NAH. In NAHGO, the small tube-like structures of 245-342 nm are distributed among the layered sheets of GO. The formation of needle like morphology is likely due to the distortion of sheets or to the impurities in the sample. The incorporation of nitrogen moieties delaminates the sheet structure with significant disorder in the graphitic structure of GO. The reduction and the integration of nitrogen in GO enhance ion transport, suitable for electrochemical sensing applications. 

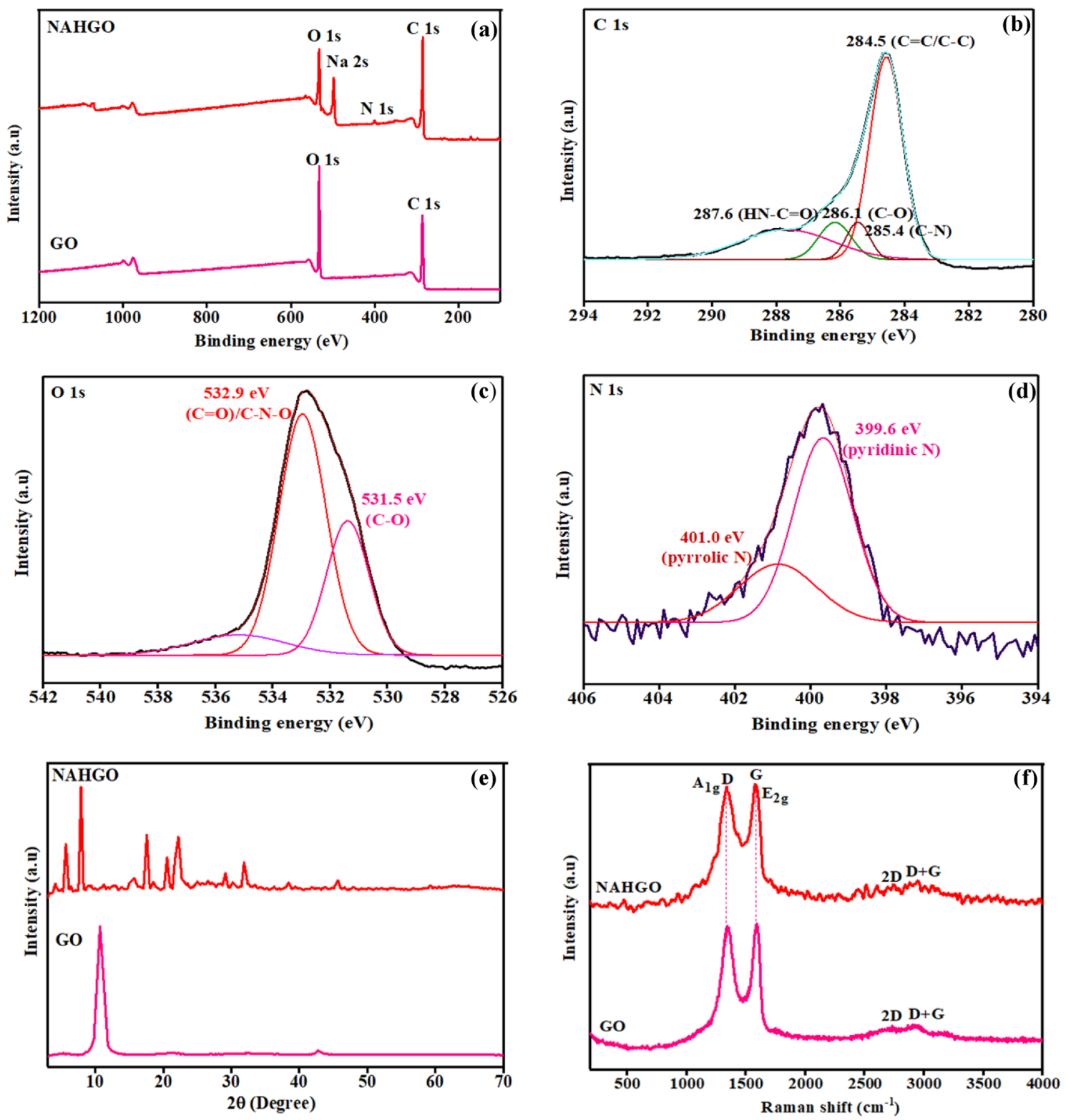

Figure 1. (a) XPS wide scan spectra of GO and NAHGO, deconvoluted peaks of (b) C1s, (c) O1s, and (d) N1s (e) Powder XRD patterns, and (f) Raman spectra of GO and NAHGO.

Electrochemical activity of NAHGO. The surface area of GO and NAHGO modified working electrodes were probed using $0.001 \mathrm{M} \mathrm{K}_{4} \mathrm{Fe}(\mathrm{CN})_{6}$ in $0.1 \mathrm{M} \mathrm{KCl}$ at a scan rate of $50 \mathrm{mV} \mathrm{s}^{-1}$ in a potential window of -0.2 to $0.8 \mathrm{~V}$, and are shown in Fig. 3a. The poor electrochemical response of $\mathrm{K}_{4} \mathrm{Fe}(\mathrm{CN})_{6}$ in $0.1 \mathrm{M} \mathrm{KCl}$ at bare GCE implies a slow transfer of electrons at the surface. The slight increase of redox peak currents at GCE modified NAHGO than GO, possibly due to fast electron transfer and the large electroactive surface area at NAHGO. This is effective in offering more active sites for caffeine oxidation. The scan rates of NAHGO in a mixture of $0.001 \mathrm{M}$ $\mathrm{K}_{4} \mathrm{Fe}(\mathrm{CN})_{6}$ and $0.1 \mathrm{M} \mathrm{KCl}$ and plots of anodic current (Ipa) and cathodic current (Ipc) vs. square root of the scan rate $\left(v^{1 / 2}\left(\mathrm{mVs}^{-1}\right)^{1 / 2}\right.$ (Figs. S3 and S4a,b) were observed. The electrochemically active surface areas of all the GCE modified electrodes were calculated with the individual slopes of the plot using Randles Sevick equation

$$
\mathrm{I}_{\mathrm{p}}=\left(2.69 \times 10^{5}\right) \mathrm{n}^{3 / 2} \mathrm{D}^{1 / 2} v^{1 / 2} \mathrm{AC}^{*}
$$

which relates the peak current of the active species with the surface area for a reversible process ${ }^{44}$. $\mathrm{I}_{\mathrm{p}}$ refers to the anodic peak current, $\mathrm{n}$ is the total number of electrons transferred $(\mathrm{n}=1), \mathrm{A}$ is the effective surface area of the electrode, $\mathrm{D}$ is the diffusion coefficient of $\mathrm{K}_{4} \mathrm{Fe}(\mathrm{CN})_{6}, \mathrm{C}^{*}$ is the concentration of $\mathrm{K}_{4} \mathrm{Fe}(\mathrm{CN})_{6}$, and $v$ is the scan rate. The calculated electroactive surface areas for bare GCE, GO, and NAHGO modified electrodes are $0.078 \mathrm{~cm}^{2}$, $0.126 \mathrm{~cm}^{2}$, and $0.29 \mathrm{~cm}^{2}$, respectively. NAHGO seemed to provide an enhanced active surface area, presumably owing to the defects in GO with electron-rich nitrogen moieties ${ }^{45}$. 

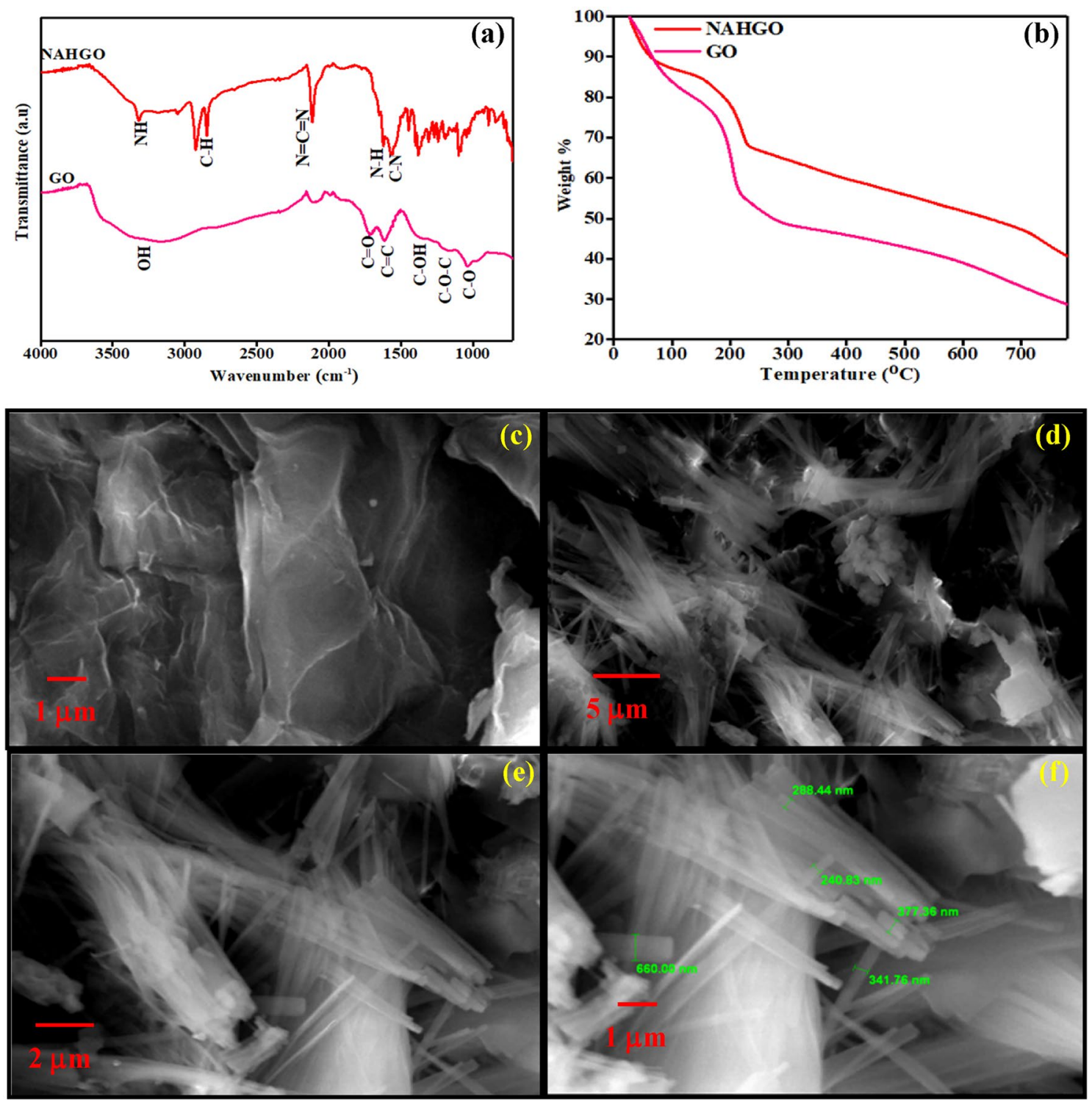

Figure 2. (a) FTIR spectra, (b) Thermogravimetric analysis, (c) SEM images of GO, and (d-f) NAHGO in different magnifications.

Electrocatalytic characteristics of NAHGO for the oxidation of caffeine. The electrochemical activities of bare GCE, GO, and NAHGO were further investigated in the presence of caffeine. Figure $3 \mathrm{~b}$ shows CVs of bare GCE, GO, and NAHGO modified GCE electrodes, at pH 2 with $100 \times 10^{-6} \mathrm{M}$ of caffeine, at a scan rate of $50 \mathrm{mV} \mathrm{s}^{-1}$. The electrocatalytic behaviour of the NAHGO was also examined in the absence and presence of caffeine. It is shown in Fig. 3c. The caffeine oxidation peak in $1.5 \mathrm{~V}$ indicates the oxidation of caffeine on NAHGO modified GCE, according to the previously reported results ${ }^{31,46}$. Caffeine has a tertiary amine group with three alkyl groups and an amide group in which a carboxyl group is connected to a nitrogen atom. In addition to these groups, imine, carboxyl, methyl, and alkene groups are also present in caffeine. The carbon-oxygen and carbon-nitrogen covalent bonds make caffeine more polar due to its molecular geometry. The distinct positive and negative parts created by the overall molecular dipole of caffeine have a strong affinity to the ends of other oppositely charged ends of the NAHGO by intermolecular attractions. Though the three nitrogen atoms of caffeine are methylated (N1, N3, and N7), as seen in Fig. S5, caffeine serves as a hydrogen bond acceptor with the ring nitrogen atom (N9) and carbonyl oxygen atoms (O2 and O6) ${ }^{47}$. Therefore the interaction of hydrogen is highly dominated by these positions of caffeine. The effective probes of interactions in NAHGO are epoxide, alcohol, carboxylic acid, pyrrolic, and pyridinic nitrogen moieties ${ }^{48}$. The alcohol and carboxylic acid oxygen containing functional groups and the $\mathrm{NH}$ of pyrrolic nitrogen can act as hydrogen bond donors and acceptors. In this case, they play the role of hydrogen donor and make electrostatic interactions with caffeine. Hence, the increased electrochemical response of NAHGO arises from the efficient interactions of highly polar functional groups of caffeine and NAHGO, which generate charge accumulations on highly polar functional groups turned out to create electrostatic interactions. These interactions are less in GO due to the fewer heteroatoms, which leads to not as much current response of NAHGO. 

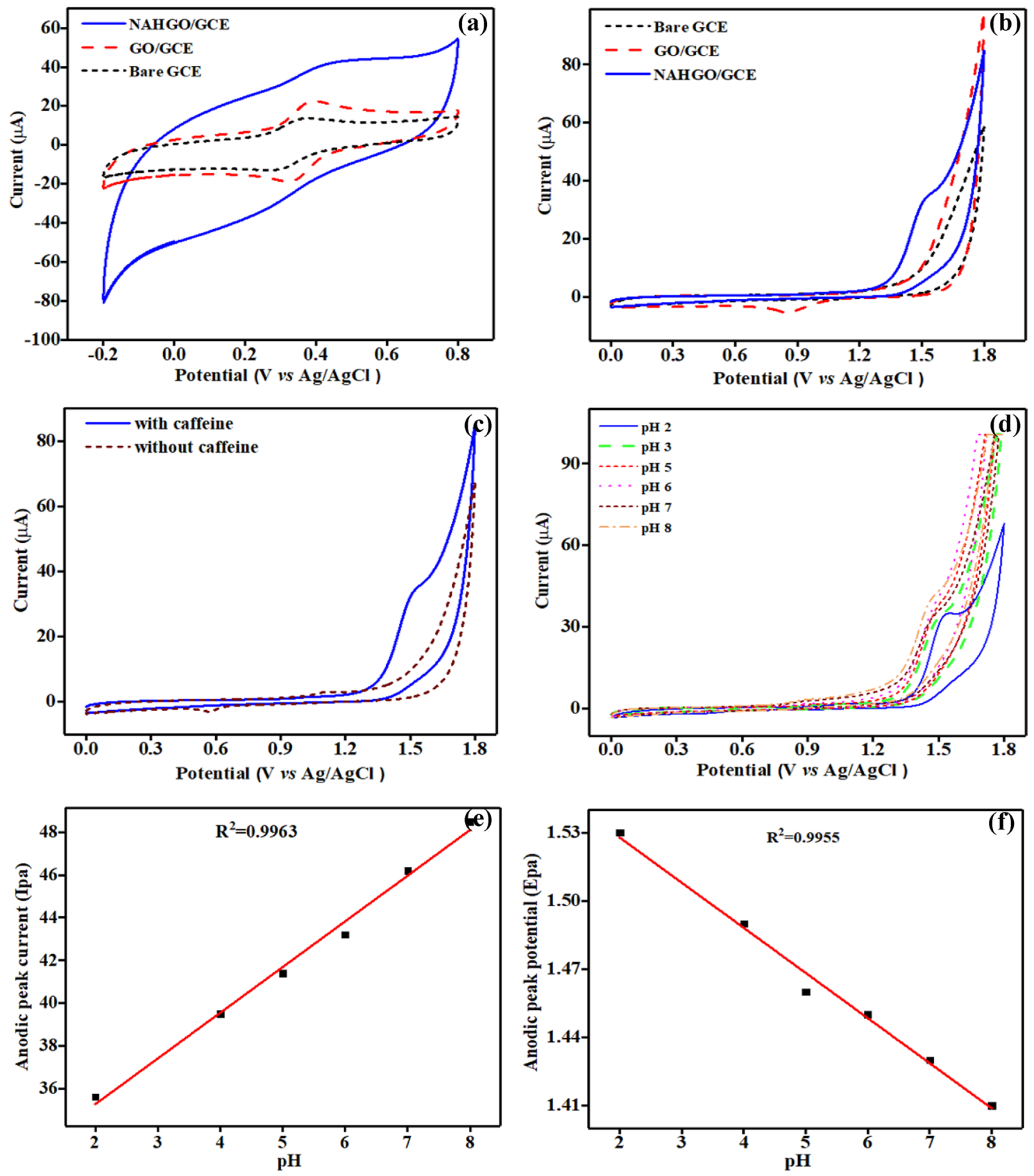

Figure 3. Cyclic voltammograms of the bare GCE, GO, and NAHGO (a) in the presence of $0.001 \mathrm{M} \mathrm{K}_{4} \mathrm{Fe}(\mathrm{CN})_{6}$ in $0.1 \mathrm{M} \mathrm{KCl},(\mathbf{b})$ at $\mathrm{pH} 2$ with $100 \times 10^{-6} \mathrm{M}$ of caffeine at a scan rate of $50 \mathrm{mV} \mathrm{s}^{-1}$, Cyclic voltammograms of NAHGO (c) with and without caffeine, (d) at $\mathrm{pH}$ values of $2,3,5,6,7,8$, in $100 \times 10^{-6} \mathrm{M}$ of caffeine, at a scan rate of $50 \mathrm{mVs}^{-1}$, Calibration plot of (e) $\mathrm{pH}$ vs. anodic current (Ipa), and (f) pH vs. anodic peak potential (Epa).

Effect of supporting electrolytes. Different electrolyte solutions of $\mathrm{pH}$ were used to understand the effect of supporting electrolyte on the cyclic voltammetric behaviour of caffeine. The effect of $\mathrm{pH}$ on the peak currents and peak potentials of caffeine electro-oxidation $\left(100 \times 10^{-6} \mathrm{M}\right)$ by NAHGO electrode was studied and is represented in Fig. 3d. Though pH 8 has a higher peak current and lower anodic peak potential for the caffeine oxidation at $\mathrm{pH} 8$, the oxidation peak current is not as sharp as $\mathrm{pH} 2$. It is also reported earlier that $\mathrm{pH} 2$ (acidic medium) is best to occur the oxidation of caffeine $e^{27,28,30}$. So, further electrochemical studies are performed at $\mathrm{pH}$ 2. The linear dependence of anodic peak current and anodic peak potential with $\mathrm{pH}$ for caffeine is shown in Fig. 3e,f. The corresponding regression equations are: $\mathrm{Ipa}=0.214 \mathrm{pH}+0.307(\mathrm{R}=0.9963)$ and $\mathrm{Epa}=2.56-0.0491$ $\mathrm{pH}(\mathrm{R}=0.9955)$. This implies that the electrochemical oxidation occurs with an equal number of proton and electron transfer process ${ }^{49}$. In the presence of acidic $\mathrm{pH}$, the exchange of electrons between the $\pi$ bonds of the graphene sheet and the lone pair of electrons bring the polarization effect at the electrode surface. The interactions of NAHGO with acidic protons are shown in Fig. 4. 


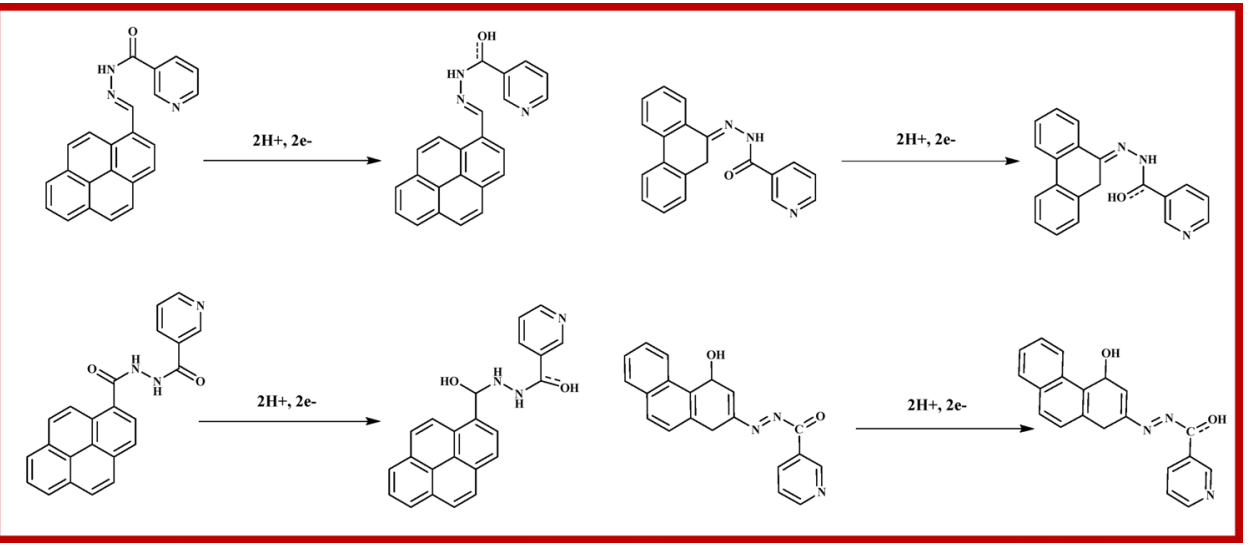

Figure 4. The interactions of NAHGO at $\mathrm{pH} 2$.

Effect of scan rate. To obtain more knowledge about the adsorption properties of NAHGO, the CVs of $100 \times 10^{-6} \mathrm{M}$ caffeine on NAHGO at various scan rates (Fig. S6) were studied for the electro-oxidation process of caffeine. As displayed in Fig. 5a, caffeine's anodic peak current increases linearly with the increase of scan rate from 10 to $60 \mathrm{mV} \mathrm{s}^{-1}$. The linear relationship of the oxidation current and the square root of the scan rates presents that the electrochemical reactions on the proposed electrode are diffusion controlled. The oxidation peak potential (Epa) shifts to more positive potential as the gradual increase of scan rates. Laviron model was used to describe the kinetic parameters of the electrochemical oxidation of caffeine ${ }^{50}$. The linear relationship of anodic oxidation potential with logarithms of scan rate is illustrated by this method. According to Laviron, the correlation between the scan rate and the oxidation peak potential is explained as follows:

$$
E p a=E^{0}+2.3 R T /[(1-\infty) n F] \$ \log v
$$

As shown in Fig. 5b, Epa exhibits the linear dependence with logarithms of scan rate (log $v)$, and the equation for linearity is Epa $=1.4326+0.0507 \log v$ with $\mathrm{R}^{2}=0.9965$. The electrochemical transmission coefficient of an irreversible process is about $0.4-0.6$, and for those processes, the value is considered to be $0.5^{51}$. Accordingly, the electronic transmission number $(\mathrm{n})$ of caffeine's electrochemical oxidation is calculated as 3.85 , which is in good agreement with the previous results ${ }^{52}$. The mechanism of electrochemical oxidation of caffeine is shown in Fig. 6, as reported earlier ${ }^{53}$. Substituted uric acid is formed in the first step, by the oxidation of two electrons and two protons at C-8-N-9 position, followed by the formation of 4,5 diol of uric acid analogue through the primary two electrons and two proton oxidation.

Analytical performance. The differential pulse voltammetric method was found to be a more sensitive method in the present work, as it produced better electrochemical performance indicators than the CV technique. Therefore, in order to achieve the favourable analytical performance using the differential pulse voltammetric (DPV) method, the parameters such as pulse amplitude (from 0.05 to $0.15 \mathrm{~V}$ ), pulse width (from 0.005 to $0.5 \mathrm{~s}$ ), and pulse period (0.1-3 s) is optimised. The best oxidation peak of caffeine was recorded as follows: pulse amplitude $0.05 \mathrm{~V}$, pulse width $0.06 \mathrm{~s}$, and pulse period of $0.5 \mathrm{~s}$ with NAHGO modified electrode in $0.1 \mathrm{M} \mathrm{H}_{2} \mathrm{SO}_{4}$ at $\mathrm{pH} 2$ in caffeine. Different concentrations from 0.01 to 40 of caffeine solutions were prepared to examine the relationship between the peak current and caffeine concentration. Figure $5 \mathrm{c}$ displays the linear increase of the anodic peak current (Ipa) with concentrations of caffeine. The calibration graph (Fig. 5d) for caffeine at NAHGO obeys the linear regression equation: Ipa $(\mu \mathrm{A})=6.883+0.0989 \mathrm{C} ; \mathrm{R}^{2}=0.9906$. The limit of detection (LOD) of caffeine is calculated to estimate this method for the determination of caffeine using the formula $3.3 \sigma / \mathrm{S}$, where $\sigma$ represents residual standard deviation and $S$ is the slope of the calibration curve ${ }^{54}$ and the LOD for NAHGO modified electrode is found to be $8.7 \times 10^{-9} \mathrm{M}$, the best reported so far. Moreover, it is compared with other reported values for the determination of caffeine using electrochemical techniques. They are presented in Table 1 and which shows the effectiveness of the proposed NAHGO sensor.

Determination of caffeine in an energy drink and pharmaceutical sample. Finally, to estimate the practical application of this method, NAHGO/GCE was used to determine caffeine in imol plus and O'cean one8 energy drink. The energy drink was degasified by ultrasonication for $10 \mathrm{~min}$ before the analysis and diluted with supporting electrolyte $\left(100 \times 10^{-6} \mathrm{M}\right)$. The tablet was grounded with mortar to a fine powder, dissolved in supporting electrolyte $\left(100 \times 10^{-6} \mathrm{M}\right)$, and sonicated for $10 \mathrm{~min}$. Subsequently, the appropriate amount of the resulting dispersion was selected and centrifuged. $9 \mathrm{~cm}^{3}$ of $0.1 \mathrm{~mol} \mathrm{~L}-1 \mathrm{pH} 2$ solution was added to $1 \mathrm{~cm}^{3}$ of the sample solution, and recorded the DPVs (Fig. 5e,f). The mean time to calculate the recovery standard solutions of caffeine is added and represented in Table S1. The recovery range of 94.9-101.9\% suggests that NAHGO is adequate for practical sensing applications. 

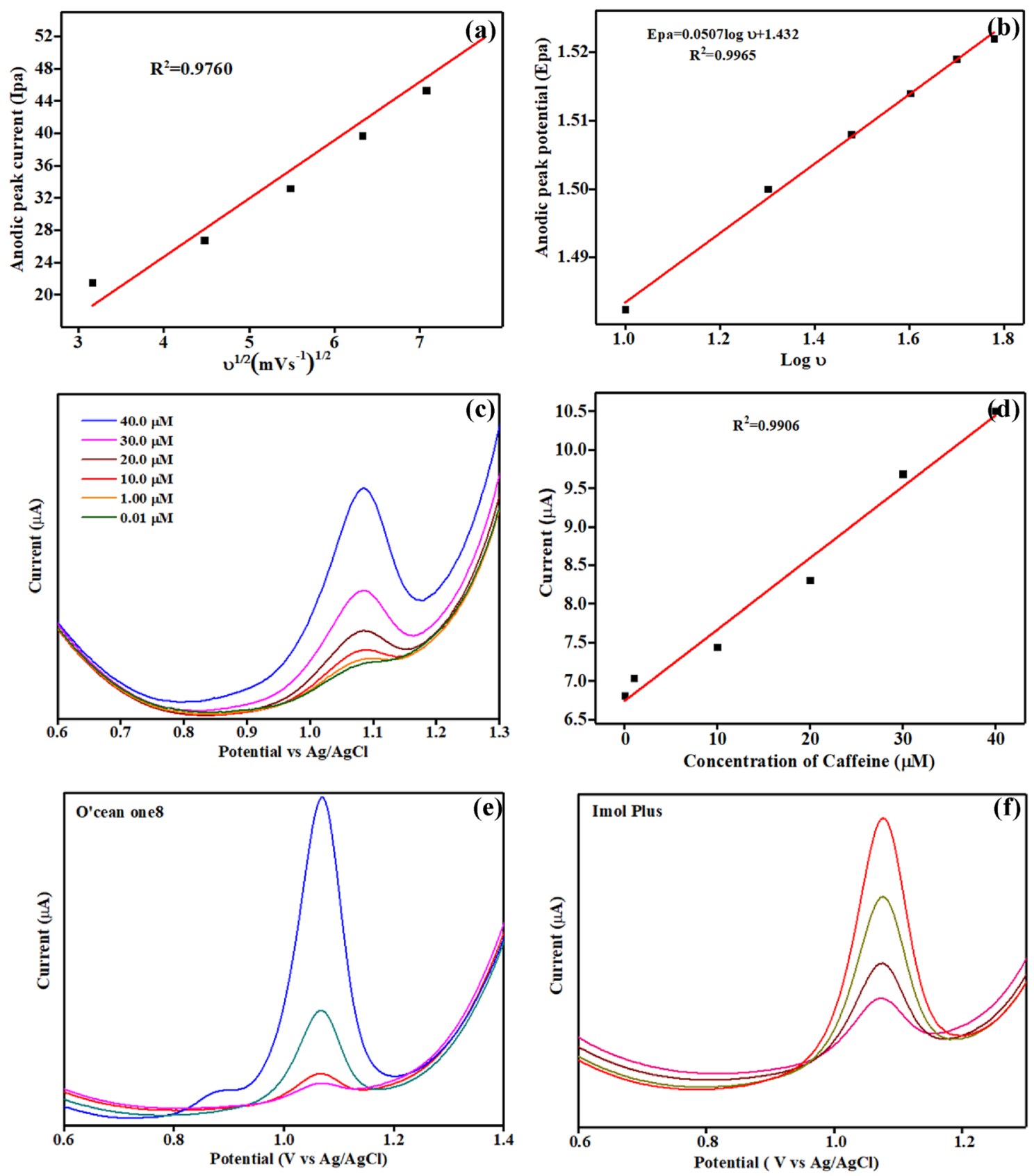

Figure 5. Calibration plot of (a) the square root of the scan rate $\left(v^{1 / 2}\right) v s$. anodic peak current (Ipa) and (b) logarithm of scan rate (log v) vs. anodic peak potential (Epa), (c) Differential pulse voltammogram (DPV) of the NAHGO modified GCE electrodes at a $\mathrm{pH}$ value of 2 with standard additions of caffeine in the order $0.01 \times 10^{-6} \mathrm{M}, 1.0 \times 10^{-6} \mathrm{M}, 10 \times 10^{-6} \mathrm{M}, 20 \times 10^{-6} \mathrm{M}, 30 \times 10^{-6} \mathrm{M}, 40 \times 10^{-6} \mathrm{M}(\mathrm{d})$ Calibration plot of caffeine concentration $(\mu \mathrm{M})$ vs. anodic peak current (Ipa), Differential pulse voltammogram of the NAHGO in (e) O'cean one 8 energy drink and (f) imol plus.

\section{Experimental}

Materials. Graphite, potassium permanganate, sulphuric acid (36 M), hydrochloric acid (5\%), sodium hydroxide, hydrogen peroxide (30\%), hydroxy benzotriazole, dicyclohexyl carbodiimide, nicotinic acid hydrazide, potassium chloride, potassium ferrocyanide, citric acid, sodium citrate, caffeine powder, potassium dihydrogen phosphate, dipotassium hydrogen phosphate were purchased from Sigma Aldrich. All the reactions were carried out under a nitrogen atmosphere with the use of the standard Schlenk technique. All the solvents and reagents were of analytical grade and used without further purification. The O'cean one8 energy drink and imol plus were purchased from a local market and pharmaceuticals, respectively. The stock solutions of caffeine $\left(100 \times 10^{-6} \mathrm{M}\right)$ were prepared fresh each time of experiments. Aqueous solutions of different $\mathrm{pH}$ of buffers were prepared, diluted using millipore water. 


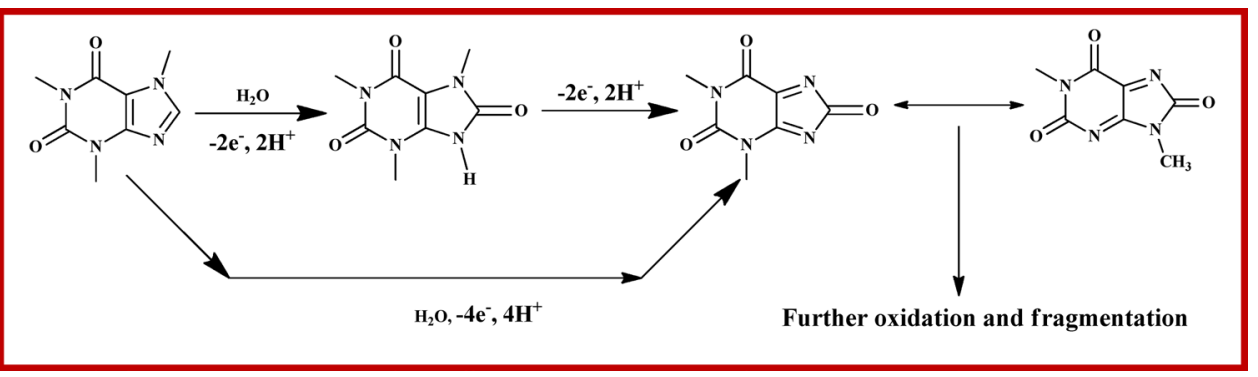

Figure 6. Mechanism of the electrochemical oxidation of caffeine at NAHGO.

\begin{tabular}{|l|l|l|l|l|}
\hline Modified electrodes & Technique & Linear range $\left(\mathbf{1 0}^{-\mathbf{6}} \mathbf{M}\right)$ & Detection limit $\left(\mathbf{1 0}^{-\mathbf{6}} \mathbf{M}\right)$ & References \\
\hline BQ/CPE & SWV & $500-8000$ & 51.00 & 54 \\
\hline Bi-CNT/CPE & SWV & $51.03-1026$ & 0.182 & 55 \\
\hline DNA/CNT/CPE & SWV & $0.5120-61.70$ & 0.350 & 56 \\
\hline MIP/CPE & DPV & $0.0600-25.00$ & 0.015 & 57 \\
\hline CTAB/GR/GCE & DPV & $0.300-100.0$ & 0.091 & 58 \\
\hline SWCNT/CPE & DPV & $0.250-100.0$ & 0.120 & 59 \\
\hline PT/TiO_-GR/GCE & DPV & $25.00-200.0$ & 0.500 & 60 \\
\hline Nitrogen doped graphene & SWV & $0.060-50.00$ & 0.020 & 61 \\
\hline Poly(Alizarin Red S)/GCE & SWV & $0.500-250.0$ & 0.060 & 62 \\
\hline Attapulgite/Nafion/GCE & DPV & $1.000-4.000$ & 0.040 & 28 \\
\hline NAHGO/GCE & DPV & $0.010-40.00$ & 0.008 & This work \\
\hline
\end{tabular}

Table 1. An overview of electrochemical methods for the determination of caffeine.

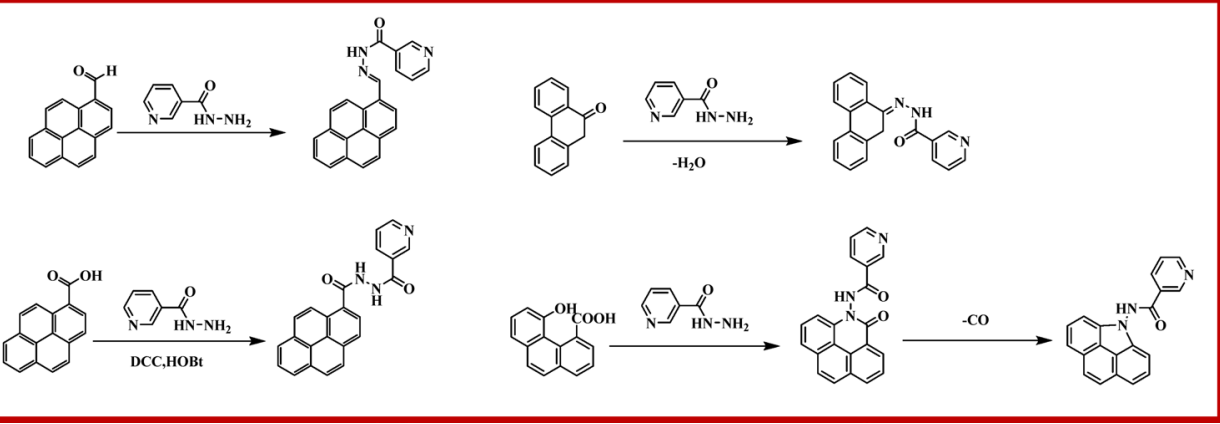

Figure 7. Reactions occur during the synthesis of NAHGO.

Synthesis of nicotinic acid hydrazide anchored graphene oxide (NAHGO). Graphene oxide (GO) was synthesised from graphite by modified Hummers method ${ }^{63}$. The nicotinic acid hydrazide anchored graphene oxide (NAHGO) was synthesised by the dispersion of GO (0.2 g) in $20 \mathrm{~cm}^{3}$ dimethylformamide (DMF) by ultrasonication method. To the solution, sodium hydroxide pellets $(0.2 \mathrm{~g}, 5.0 \mathrm{mmol})$ were added, and the resulting solution was stirred for $60 \mathrm{~min}$ at room temperature. Consequently, nicotinic acid hydrazide $(0.2 \mathrm{~g}$, $1.45 \mathrm{mmol})$, hydroxybenzotriazole $(0.2 \mathrm{~g}, 2.81 \mathrm{mmol})$ followed by dicyclohexylcarbodiimide $(0.2 \mathrm{~g}, 2.66 \mathrm{mmol})$ were added to the above reaction mixture and stirred for $24 \mathrm{~h}$ in nitrogen atmosphere. The resultant NAHGO powder collected by centrifugation was added to pure DMF and the resulting suspension was again centrifuged to remove the side products. This process was repeated twice with DMF and then with water to remove DMF to give pure NAHGO. The NAHGO prepared was dried overnight at $60^{\circ} \mathrm{C}$. The amide, amine, and pyridinic groups are present in NAHGO. The reaction of the amino group of NAH with the hydroxyl of carboxylic acid generates another amide bond in NAHGO. The imine can form with the amine group of $\mathrm{NAH}$ and the $-\mathrm{C}=\mathrm{O}$ or $-\mathrm{HC}=\mathrm{O}$ group of GO. The nearby carboxylic acid and hydroxyl groups can undergo an esterification reaction followed by the generation of a pyrrole ring. Hence we believe that the treatment of NAH in GO creates more defects due to the reduction of GO with nitrogen doping. The suggested reactions which occur during the synthesis of NAHGO are shown in Fig. 7. 


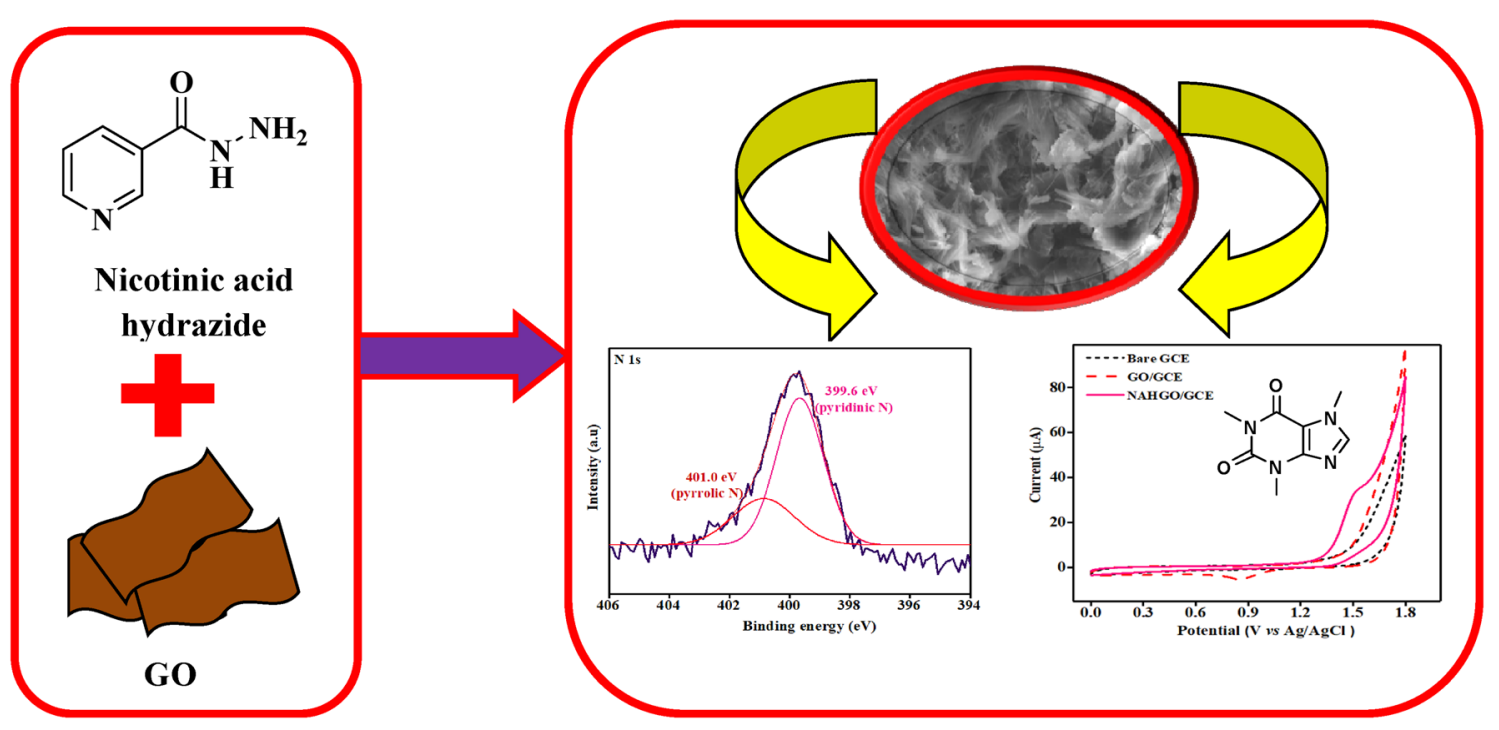

Figure 8. Synthesis and electrochemical activity of NAHGO as a sensor.

Characterisation techniques. The characteristic functional groups of different samples were recorded using an Agilent technologies FTIR spectrometer in the range $400-4000 \mathrm{~cm}^{-1}$. Powder X-ray Diffraction (P-XRD) patterns were carried out in a Brucker AXSD8 Advance using Ni-filtered $\mathrm{Cu}-\mathrm{K}_{\alpha} \mathrm{X}$-ray source $(\lambda=1.5406 \AA)$, with a scan speed of $2^{\circ} \mathrm{min}^{-1}$. Thermogravimetric analysis (TGA) was recorded in Perkin Elmer STA 6000. The composition and disorders of anchored compounds were analyzed by X-ray photoelectron spectroscopy (Thermoscientific K-alpha surface analyser). Raman spectra of GO and NAHGO were recorded in HORIBA Jobin Yvon Lab RAM HR 800, equipped with a thermoelectrically cooled CCD detector. The morphology and size of GO and NAHGO were recorded on a JEOL Model JSM-6390LV scanning electron microscope. Cyclic voltammetric (CV) and differential pulse voltammetric (DPV) measurements were carried out on a CHI608E electrochemical workstation with a three-electrode system saturated $\mathrm{Ag} / \mathrm{AgCl}$ acted as the reference electrode, $\mathrm{Pt}$ wire as a counter electrode, and the modified glassy carbon electrode as the working electrode. The preparation of the NAHGO modified glassy carbon electrode and the different buffers used are given in supporting information. Synthesis and electrochemical activity of NAHGO as a sensor are shown in Fig. 8.

\section{Conclusion}

In summary, the synthesis and properties of NAHGO for electrochemical detection of caffeine were discussed. The structure and morphologies of NAHGO were studied by IR, P-XRD, Raman, SEM, and TGA. The electrochemical oxidation of NAHGO towards the alkaloid caffeine was analyzed. NAHGO displayed enhanced electrochemical activity towards the determination of caffeine, with an improved detection limit of $8.7 \times 10^{-9} \mathrm{M}$. The NAHGO sensor was also employed to determine caffeine in Imol plus and O'cean one8 energy drink. Electrochemical studies exhibited that the NAHGO can be used as an efficient and reliable analytical tool for caffeine detection.

Received: 5 December 2020; Accepted: 5 April 2021

Published online: 03 June 2021

\section{References}

1. Elham, A., Masoumeh, G. \& Saeed, S. Electrochemical sensing based on carbon nanoparticles: A review. Sens. Actuators B Chem. 293, 183-209 (2019)

2. Bottari, G., Ángeles, H. M. \& Wibmer, L. Chemical functionalization and characterization of graphene-based materials. Chem. Soc. Rev. 46, 4464-4500 (2017).

3. Yu, W., Sisi, L., Haiyan, Y. \& Jie, L. Progress in the functional modification of graphene/graphene oxide: A review. RSC Adv. 10, 15328-15345 (2020).

4. Jose, J. et al. Photophysical and electrochemical studies of anchored chromium (III) complex on reduced graphene oxide via diazonium chemistry. Appl. Organomet. Chem. 33, e5063 (2019).

5. Yadav, R. \& Dixit, C. K. Synthesis, characterization and prospective applications of nitrogen-doped graphene: A short review. J. Sci. Adv. Mater. Devices 2, 141-149 (2017).

6. Jose, J. et al. Emerging ternary nanocomposite of $\mathrm{rGO}$ draped palladium oxide/polypyrrole for high performance supercapacitors. J. Alloys Compd. 855, 157481 (2021).

7. Geng, D. et al. Nitrogen doping effects on the structure of graphene. Appl. Surf. Sci. 257, 9193-9198 (2011).

8. Gong, K., Du, F., Xia, Z., Durstock, M. \& Dai, L. Nitrogen-doped carbon nanotube arrays with high electrocatalytic activity for oxygen reduction. Science 323, 760-764 (2009).

9. Cai, Z. et al. Synthesis of nitrogen-doped graphene quantum dots at low temperature for electrochemical sensing trinitrotoluene. Anal. Chem. 87, 11803-11811 (2015).

10. Jose, J., Jose, S. P., Abinaya, S., Shaji, S. \& Sreeja, P. B. Benzoyl hydrazine anchored graphene oxide as supercapacitor electrodes. Mat. Chem. Phys. 256, 123666 (2020). 
11. Santanu, S., Elena, B. \& Haddon, R. C. Covalent chemistry in graphene electronics. Mater. Today 15, 276-285 (2012).

12. Kemp, K. C., Georgakilas, V., Otyepka, M., Bourlinos, A. B. \& Chandra, V. Functionalization of graphene: Covalent and noncovalent approaches, derivatives and applications. Chem. Rev. 112, 6156-6214 (2012)

13. Mejia, E. G. \& Ramirez-Mares, M. V. Impact of caffeine and coffee on our health. Trends Endocrin. Met. 25, 489-492 (2014).

14. Juliana, D. \& Farah, A. Caffeine consumption through coffee: Content in the beverage, metabolism, health benefits and risks. Beverages 5, 37 (2019).

15. Durrant, K. L. Known and hidden sources of caffeine in drug, food, and natural products. J. Am. Pharm. Assoc. 42, 625-637 (2002).

16. Robin, P. et al. Coffee consumption and health: Umbrella review of meta-analyses of multiple health outcomes. BMJ 359, j5024 (2017).

17. Heaney, R. P. Effects of caffeine on bone and the calcium economy. Food Chem. Toxicol. 40, 1263-1270 (2002).

18. Poiger, I. J. B. T., Müller, M. D. \& Buser, H. R. Caffeine, an anthropogenic marker for wastewater contamination of surface waters. Environ. Sci. Technol. 37, 691-700 (2003).

19. Senta, I., Lor, E. G., Borsotti, A., Zuccato, E. \& Castiglioni, S. Wastewater analysis to monitor use of caffeine and nicotine and evaluation of their metabolites as biomarkers for population size assessment. Water Res. 74, 23-33 (2015).

20. Brodin, T. et al. Ecological effects of pharmaceuticals in aquatic systems-impacts through behavioural alterations. Philos. Trans. R Soc. Lond. B Biol. Sci. 369, 20130580 (2014).

21. Eisele, A. P. P., Valezia, C. F. \& Sartori, E. R. Exploiting the high oxidation potential of carisoprodol on a boron-doped diamond electrode: An improved method for its simultaneous determination with acetaminophen and caffeine. Analyst 142, 3514-3521 (2017).

22. Reskety, A. A., Chamjangali, M. A., Boujnane, M. \& Brajter-Tot, A. High sensitivity and fast oxidation of caffeine in coffee and theophylline at nanostructured electrodes. Electroanalysis 28, 1-9 (2016).

23. Oren, T. \& Anık, U. Voltammetric determination of caffeine by using gold nanoparticle-glassy carbon paste composite electrode. Measurement 106, 26-30 (2017).

24. Svorc, L. Determination of caffeine: A comprehensive review on electrochemical methods. Int. J. Electrochem. Sci. 8, 5755-5773 (2013).

25. Ramachandran, R. et al. A review of the advanced developments of electrochemical sensors for the detection of toxic and bioactive molecules. Inorg. Chem. Front. 6, 3418-3439 (2019).

26. Kalita, G. \& Tanemura, M. Fundamentals of chemical vapor deposited graphene and emerging applications. In Graphene MaterialsAdvanced Applications (eds George, K. \& Athanasios, M.) 30-35 (IntechOpen, 2017).

27. Wang, Y., Wei, X., Wang, F. \& Li, M. Sensitive voltammetric detection of caffeine in tea and other beverages based on a DNA functionalized single-walled carbon nanotube modified glassy carbon electrode. Anal. Methods 6, 7525-7531 (2014).

28. Tajeu, K. Y., Ymele, E., Jiokeng, S. L. Z. \& Tonle, I. K. Electrochemical sensor for caffeine based on a glassy carbon electrode modified with an Attapulgite/nafion Film. Electroanalysis 31, 350-356 (2018).

29. Trani, A., Petrucci, R., Marrosu, G., Zane, D. \& Curulli, A. Selective electrochemical determination of caffeine at a gold chitosan nanocomposite sensor: May little change on nanocomposites synthesis affect selectivity. J. Electroanal. Chem. 788, 99-106 (2017).

30. Silva, T., Zanin, H., Corat, E. J. \& Fatibello-Filho, O. Simultaneous voltammetric determination of paracetamol, codeine and caffeine on diamond-like carbon porous electrodes. Electroanalysis 29, 907-916 (2017).

31. Shehata, M., Azabb, S. M. \& Fekry, A. M. May glutathione and graphene oxide enhance the electrochemical detection of caffeine on carbon paste sensor in aqueous and surfactant media for beverages analysis?. Synth. Met. 256, 116122 (2019).

32. Zhang, Y. et al. Phenolic hydroxyl functionalized partially reduced graphene oxides for symmetric supercapacitors with significantly enhanced electrochemical performance. J. Power Sources 415, 226779 (2019).

33. Precht, R. et al. Investigation of sodium insertion into tetracyanoquinodimethane (TCNQ): Results for a TCNQ thin film obtained by a surface science approach. Phys. Chem. Chem. Phys. 4, 3056-3064 (2016).

34. Savintsev, A. P., Yu, O. G., Kalazhokov, Z. K. \& Kalazhokov, K. K. X-ray photoelectron spectroscopy studies of the sodium chloride surface after laser exposure. J. Phys. Conf. Ser. 774, 012118 (2016).

35. Jhonsi, M. A., Nithya, C. \& Kathiravan, A. Probing electron transfer dynamics of pyranine with reduced graphene oxide. Phys. Chem. Chem. Phys. 16, 20878-20886 (2014).

36. Shanshan, W., Yang, L., Xiaobin, F., Fengbao, Z. \& Guoliang, Z. $\beta$-Cyclodextrin functionalized graphene oxide: An efficient and recyclable adsorbent for the removal of dye pollutants. Front. Chem. Sci. Eng. 9, 77-83 (2015).

37. Kumari, S., Shekhar, A. \& Pathak, D. D. Synthesis and characterization of a $\mathrm{Cu}$ (II) Schiff base complex immobilized on graphene oxide and its catalytic application in the green synthesis of propargylamines. RSC Adv. 6, 15340-15344 (2016).

38. Mungse, H. P., Verma, S., Kumar, N., Sain, B. \& Khatri, O. P. Grafting of oxo-vanadium Schiff base on graphene nanosheets and its catalytic activity for the oxidation of alcohols. J. Mater. Chem. 22, 5427-5433 (2012).

39. Chakraborty, S. et al. High yield synthesis of amine functionalized graphene oxide and its surface properties. RSC Adv. 6, 6791667924 (2016).

40. Jia, Y. A. N. et al. Functionalized graphene oxide with ethylenediamine and 1,6-hexane diamine. New Carbon Mater. 27, 370-376 (2012).

41. Anil, K. \& Mahima, K. Amino acid mediated functionalization andreduction of graphene oxide-synthesis and the formation mechanism of nitrogen-doped graphene. New. J. Chem. 38, 3457-3467 (2014).

42. Yake, Z. et al. Nitrogen-doped graphene as a cathode material for dye-sensitized solar cells: Effects of hydrothermal reaction and annealing on electrocatalytic performance. RSC Adv. 5, 10430-10439 (2015).

43. Jiao, Z. et al. 3D free-standing nitrogen-doped reduced graphene oxide aerogel as anode material for sodium ion batteries with enhanced sodium storage. Sci. Rep. 7, 4886 (2017).

44. Allen J. B., Larry, R., Faulkner, Electrochemical Methods: Fundamentals and Applications, John Wiley \& Sons Inc. 2nd Edn., 261-304 (2000)

45. Choi, S., Kim, C., Suh, J. M. \& Jang, H. W. Reduced graphene oxide-based materials for electrochemical energy conversion reactions. Carbon Energy 1, 85-108 (2019).

46. Arroyo-Gomez, J. J., Villarroel-Rocha, D., de Freitas-Araújo, K. C., Martínez-Huitle, C. A. \& Sapag, K. Applicability of activated carbon obtained from peach stone as an electrochemical sensor for detecting caffeine. J. Electroanal. Chem. 822, 171-176 (2018).

47. Tavagnacco, L. et al. Molecular dynamics simulation studies of caffeine aggregation in aqueous solution. J. Phys. Chem. B 115, 10957-10966 (2011).

48. Hezarkhani, M. \& Ghadari, R. Exploration of the binding properties of the azo dye pollutants with nitrogen-doped graphene oxide by computational modeling for wastewater treatment improvement. Chem. Select 4, 5968-5978 (2019).

49. Wang, Y. et al. An electrochemical sensor for determination of tryptophan in the presence of DA based on poly(L-methionine)/ graphene modified electrode. RSC Adv. 6, 10662-10669 (2016).

50. Wang, Y., Dinga, Y., Lia, L. \& Huc, P. Nitrogen-doped carbon nanotubes decorated poly (L-cysteine) as a novel, ultrasensitive electrochemical sensor for simultaneous determination of theophylline and caffeine. Talanta 178, 449-457 (2018).

51. Pelossof, G., Tel-Vered, R., Shimrona, S. \& Willne, I. Controlling interfacial electron transfer and electrocatalysis by $\mathrm{pH}$ or ionswitchable DNA monolayer-modified electrodes. Chem. Sci. 4, 1137-1144 (2013).

52. Spataru, N., Sarada, B. V., Tryk, D. A. \& Fujishima, A. Anodic voltammetry of xanthine, theophylline, theobromine and caffeine at conductive diamond electrodes and its analytical application. Electroanalysis 14, 721-728 (2002). 
53. Silva, A. R. L. et al. Understanding the behavior of caffeine on a boron-doped diamond surface: Voltammetric, DFT, QTAIM and ELF studies. New J. Chem. 41, 7766-7774 (2017).

54. Aklilu, M., Tessema, M. \& Redi-Abshiro, M. Indirect voltammetric determination of caffeine content in coffee using 1,4-benzoquinone modified carbon paste electrode. Talanta 76, 742-746 (2008).

55. Ly, S. Y., Jung, Y., Lee, J. \& Kwak, K. Simultaneous diagnostic assay of catechol and caffeine using an in vivo 1 mplanted neuro sensor. Bull. Kor. Chem. Soc. 29, 1742-1746 (2008).

56. Ly, S. Y., Lee, C. H. \& Jung, Y. S. Voltammetric bioassay of caffeine using sensor implant. Neuromol. Med. 11, $20-27$ (2009).

57. Alizadeh, T., Ganjali, M. R., Zare, M. \& Norouzi, P. Development of a voltammetric sensor based on a molecularly imprinted polymer (MIP) for caffeine measurement. Electrochim. Acta 55, 1568-1574 (2010).

58. Jun-Yong, S., Ke-Jing, H., Shuai-Yun, W. \& Zhi-Wei, W. Application of cetyltrimethylammonium bromide-graphene modified electrode for sensitive determination of caffeine. Can. J. Chem. 89, 697-702 (2011).

59. Habibi, B., Abazari, M. \& Pournaghi-Azar, M. H. A carbon nanotube modified electrode for determination of caffeine by differential pulse voltammetry. Chin. J. Cat. 33, 1783-1790 (2012).

60. Xiao-Qin, X., Ke-Jing, H. \& Chun-Xuan, X. Glassy carbon electrode modified with poly(taurine)/TiO ${ }_{2}$-graphene composite film for determination of acetaminophen and caffeine. Chem. Ind. Chem. Eng. 19, 359-368 (2013).

61. Jiang, L., Ding, Y., Jiang, F., Li, L. \& Mo, F. Electrodeposited nitrogen-doped graphene/carbon nanotubes nanocomposite as enhancer for simultaneous and sensitive voltammetric determination of caffeine and vanillin. Anal. Chim. Acta 833, $22-28$ (2014).

62. Filik, H., Avan, A. A. \& Mümin, Y. Simultaneous electrochemical determination of caffeine and vanillin by using poly(Alizarin Red S) modified glassy carbon electrode. Food Anal. Methods 10, 31-40 (2017).

63. Hummers, W. S. \& Offeman, R. E. Preparation of graphitic oxide. J. Am. Chem. Soc. 80, 1339 (1958).

\section{Author contributions}

Credit Author Statement: J.J.: Investigation, V.S.: Resources. S.S.: Resources, P.B.S.: Conceptualization.

\section{Competing interests}

The authors declare no competing interests.

\section{Additional information}

Supplementary Information The online version contains supplementary material available at https://doi.org/ 10.1038/s41598-021-89427-6.

Correspondence and requests for materials should be addressed to P.B.S.

Reprints and permissions information is available at www.nature.com/reprints.

Publisher's note Springer Nature remains neutral with regard to jurisdictional claims in published maps and institutional affiliations.

Open Access This article is licensed under a Creative Commons Attribution 4.0 International License, which permits use, sharing, adaptation, distribution and reproduction in any medium or format, as long as you give appropriate credit to the original author(s) and the source, provide a link to the Creative Commons licence, and indicate if changes were made. The images or other third party material in this article are included in the article's Creative Commons licence, unless indicated otherwise in a credit line to the material. If material is not included in the article's Creative Commons licence and your intended use is not permitted by statutory regulation or exceeds the permitted use, you will need to obtain permission directly from the copyright holder. To view a copy of this licence, visit http://creativecommons.org/licenses/by/4.0/.

(C) The Author(s) 2021 